\title{
Evaluation of integral forces and pressure fields from planar velocimetry data for incompressible and compressible flows
}

\author{
B. W. van Oudheusden - F. Scarano • \\ E. W. M. Roosenboom - E. W. F. Casimiri · \\ L. J. Souverein
}

Received: 28 August 2006/Revised: 9 January 2007 / Accepted: 9 January 2007 / Published online: 16 February 2007

(C) Springer-Verlag 2007

\begin{abstract}
The approach to determine pressure fields and integral loads from planar velocimetry data is discussed, in relation to the implementation for incompressible and compressible flows around twodimensional objects. The method relies upon the application of control-volume approaches in combination with the deduction of the pressure field from the experimental data, by making use of the flow constitutive equations. In this paper the implementation for two specific application areas is addressed. The first is time-mean pressure field and force evaluation from velocity ensemble statistics, as obtained from timeuncorrelated PIV acquisition, for incompressible flow. Two test cases are considered for this flow regime: the unsteady vortical flow around a square section cylinder at incidence, as well as the force characterization of a low-speed airfoil. The second topic considers the extension of the method to steady compressible flow, with the supersonic flow around a bi-convex airfoil as experimental test case. As in this flow regime the density appears as an extra unknown in the momentum equation, additional flow equations need to be invoked. A convenient approach for this was found, using the gas law and the adiabatic flow condition, with which the pressure-integration procedure becomes essentially the same as for the incompressible case.
\end{abstract}

B. W. van Oudheusden $(\bowtie) \cdot$ F. Scarano .

E. W. M. Roosenboom - E. W. F. Casimiri .

L. J. Souverein

Department of Aerospace Engineering,

Delft University of Technology,

Kluyverweg 1, 2629 HS Delft, The Netherlands

e-mail: b.w.vanoudheusden@tudelft.nl

\section{Introduction}

Many fluid dynamic applications involve configurations where relatively slender objects are exposed to a crossflow, such as is the case for aircraft wings, wind turbine blades, bridge decks, towers, etc. In such situations the flows are predominantly two-dimensional (at least in the mean sense) and are conveniently studied by means of planar velocimetry techniques such as PIV (Particle Image Velocimetry) in particular (Raffel et al. 1998; Adrian 2005). The PIV technique is a very powerful non-intrusive technique to study separated flows of high complexity and has developed into a reliable workhorse for fundamental and industrial fluid dynamics research over the last decades. It is capable of delivering instantaneous velocity field data over a complete region of interest, hence revealing and quantifying the instantaneous spatial structures of the flow.

In technical fluid-dynamic applications there is a further special interest in the loads aspects of the flow, notably pressure distributions as well as integral aerodynamic forces and moments. In current experimental research practice, flow field information and mechanical loads are obtained by separate techniques. Traditionally, surface pressure distributions are obtained by means of pressure tappings in the model (or using pressure sensitive paint) while integral loads are measured with mechanical balance systems in the model or its support. An appealing approach to establish a direct link between flow behaviour and forcing mechanisms is by deriving the loads from the flow field information itself. Apart from the inherent synchronisation between the different flow properties, it further removes the necessity of additional and/or intrusive 
instrumentation of the model itself. This makes it a very powerful and flexible tool to study flow-structure interactions at both fundamental and applied level of interest. A well-known technique in aeronautical windtunnel operations is the wake-survey method in which the drag of a wing is derived from the momentum deficit in the wake (Jones 1936; Brune 1994), which can be seen as an example of this loads determination principle established in practice. Furthermore, several procedures have been proposed recently, that allow unsteady loads to be determined from time-resolved PIV data (Lin and Rockwell 1996; Unal et al. 1997; Noca et al. 1999; Berton et al. 2004; Fujisawa et al. 2005), based on variants of the control-volume approach.

\section{Theoretical background}

The control-volume approach allows the integral load on an object to be obtained from an integration of the flow variables inside a control volume surrounding this object (Anderson 1991). Considering a fixed control volume $S$ enclosing an object as in Fig. 1, the instantaneous value of the force experienced by the object is related to the flow field variables as:

$$
\begin{aligned}
\mathbf{F}(t)= & -\iiint_{s} \frac{\partial \rho \mathbf{V}}{\partial t} \mathrm{~d} S-\iint_{s} \rho(\mathbf{V} \cdot \mathbf{n}) \mathbf{V} \mathrm{d} s \\
& +\iint_{s}(-p \mathbf{n}+\tau \cdot \mathbf{n}) \mathrm{d} s
\end{aligned}
$$

with $S$ the control volume and $s$ its outer contour with $\mathbf{n}$ the outward pointing normal. Flow field properties are the velocity $\mathbf{V}$, the pressure $p$, the density $\rho$, and the viscous stress $\tau$. The contribution of the viscous stresses can generally be neglected when the control volume contour is taken sufficiently far from the body, but may

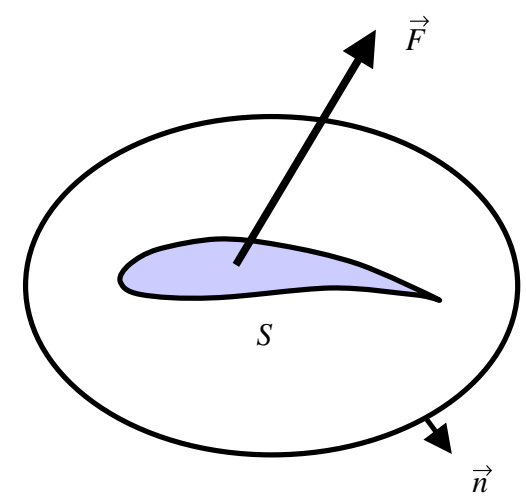

Fig. 1 Basic working principle: control-volume approach for determining integral aerodynamic forces in a two-dimensional flow configuration be included for completeness. Also the torque exerted by the aerodynamic forces can be determined from the flow field information by means of a similar integral balance procedure (Van Oudheusden et al. 2006).

Assuming incompressible flow the density is a constant and a direct application of the control-volume formulation requires the velocity and acceleration distribution inside the volume, as well as the pressure on the outer contour. The latter, however, is generally not available in a PIV experiment. Basically two approaches can be followed for this. The first is to use a formulation of the control-volume approach from which the pressure has been eliminated analytically (Noca et al. 1999). The second is to explicitly evaluate the pressure (Unal et al. 1997) using the momentum equation:

$-\nabla p=\rho \frac{\partial \mathbf{V}}{\partial t}+\rho(\mathbf{V} \cdot \nabla) \mathbf{V}-\mu \nabla^{2} \mathbf{V}$

For 2D incompressible flow the instantaneous pressure gradient can be derived directly from the velocity information, after which the pressure itself is obtained from spatial integration of the pressure gradient. Note that only the pressure on the contour is required to obtain the integrated loads. However, the pressure gradient integration may be extended to the entire flow domain of interest, using either some sort of gradient-integration scheme or through solving the Poisson equation for the pressure (Bauer and Koengeter 1999; Gurka et al. 1999; Hosokawa et al. 2003; Fujisawa et al. 2005). Special care may be needed in the processing and further conditioning of the PIV data, especially when obtained near solid surfaces, and the subsequent numerical integration process to obtain reliable and accurate pressure and load data.

\subsection{Time-mean pressure and integral loads from PIV velocity statistics}

Although these procedures in principle allow to obtain instantaneous pressure field and integral force data, practice often does not permit to perform time-resolved velocity measurements and/or to determine acceleration with a sufficient level of accuracy. Furthermore, in many fluid dynamic applications of technical interest it may be sufficient to study the flow in the mean sense, and obtaining knowledge on timemean loads. Averaging Eq. 1 in time and using tensor notation (velocity components are denoted by $u_{i}$ and averaged properties are indicated by an overbar), yields: 


$$
\begin{aligned}
\bar{F}_{i}= & -\rho \iint_{s} \bar{u}_{i} \bar{u}_{j} n_{j} \mathrm{~d} s-\rho \iint_{s} \overline{u_{i}^{\prime} u_{j}^{\prime}} n_{j} \mathrm{~d} s \\
& -\iint_{s} \bar{p} n_{i} \mathrm{~d} s+\mu \iint_{s}\left(\frac{\partial \bar{u}_{i}}{\partial x_{j}}+\frac{\partial \bar{u}_{j}}{\partial x_{i}}\right) n_{j} \mathrm{~d} s
\end{aligned}
$$

The terms on the right hand side represent the respective contributions of mean and turbulent momentum transfer, pressure and mean viscous stresses. This result shows that for the purpose of obtaining time-mean loads it is sufficient if the pressure and velocity (gradients) as well as the velocity second moments (turbulent stresses) on the outer contour of the control volume are determined. The mean pressure is obtained from averaging of the instantaneous momentum equation 2 , which yields the time-mean pressure gradient as:

$-\frac{\partial \bar{p}}{\partial x_{i}}=\rho \bar{u}_{j} \frac{\partial \bar{u}_{i}}{\partial x_{j}}+\rho \frac{\partial \overline{u_{i}^{\prime} u_{j}^{\prime}}}{\partial x_{j}}-\mu \frac{\partial^{2} \bar{u}_{i}}{\partial x_{j} \partial x_{j}}$

For 2-D flow all properties on the right-hand side can be delivered by planar velocimetry, in terms of mean values and statistics of the velocity fields. This shows how for an unsteady flow the time-mean pressure field and integral loads can be inferred from velocity field statistical data that can be obtained with standard, time-uncorrelated PIV acquisition.

\subsection{Compressible flow}

Application of the above methods to the compressible flow regime seems to have received little attention so far. Zhu et al. (2002) adapted Noca's formulations to the case of steady compressible flow and compared procedures for determining integral force from flow field data, for the numerical simulation of a transonic airfoil. The variable density, however, was taken from the simulation data as well, which cannot be done in PIV experiments where only velocity data are available.

As in the case of compressible flow the density and viscosity appear as extra unknowns in the momentum equation, additional flow equations need to be invoked. One potentially appealing approach could be to use the continuity equation to directly derive density from velocity gradients (divergence). However, this procedure exhibits some less desirable features. As the continuity equation essentially transports density information along streamlines, errors in the divergence term due to measurement inaccuracy or out-of-plane velocity gradients, accumulate in the computation. Hence, any slight three-dimensionality of the flow may seriously disrupt the density computation. Also, closed streamline regions, such as flow separation regions, cannot be approached. A more convenient approach for estimating the density was found, using the gas law and the adiabatic flow condition. The gas law is used to replace the density by pressure and temperature, while the assumption of constant total enthalpy is employed to express temperature in terms of the velocity magnitude $V$ :

$$
\frac{T}{T_{\infty}}=1+\frac{\gamma-1}{2} M_{\infty}^{2}\left(1-\frac{V^{2}}{V_{\infty}^{2}}\right)
$$

where $V_{\infty}$ and $M_{\infty}$ are the velocity and Mach number of the free stream, and $\gamma$ the ratio of specific heats $(\gamma=1.4$ for air). The adiabatic flow assumption is reasonable, also for viscous regions, in the case of steady flow without significant heat transfer (White 1991). Introducing these expressions and assuming the viscous contributions can be neglected, the momentum equation for steady flow can be written as:

$$
\begin{aligned}
\frac{-\nabla p}{p} & =-\nabla \ln \left(p / p_{\infty}\right) \\
& =\frac{\gamma M_{\infty}^{2}}{V_{\infty}^{2}+\frac{\gamma-1}{2} M_{\infty}^{2}\left(V_{\infty}^{2}-V^{2}\right)} \cdot(\mathbf{V} \cdot \nabla) \mathbf{V}
\end{aligned}
$$

This yields the pressure-integration procedure in essentially similar functional form as for the incompressible case, cf. Eq. 2, allowing the same numerical integration routines to be applied. After the pressure has been computed in this way, the density can subsequently be computed from the gas law, using the temperature from Eq. 5.

\subsection{Corrected formulation to account for mass conservation}

Especially for slender wing-like objects, the lift component of the total force is usually significantly larger than the drag component. Also, with regard to the contour integral expression of Eq. 1, the lift term is dominated by the pressure contribution and the drag by the momentum-flux term. The consequence of this is that any violation of integral mass conservation over the integration contour surface, for example due to measurement uncertainty, will be felt as an additional momentum flux and hence affects the loads determination, in particular the drag. To partly remedy this, the contour integral approach can be corrected by subtracting the force equivalent to the momentum flux generated by the net mass flux defect multiplied by the 
free stream velocity. With this, the loads estimate relation of Eq. 1 can be rewritten (assuming steady flow) as:

$$
\begin{aligned}
\mathbf{F}= & \iint_{s} \rho(\mathbf{V} \cdot \mathbf{n})\left(\mathbf{V}_{\infty}-\mathbf{V}\right) \mathrm{d} s \\
& +\iint_{s}\left(\left(p_{\infty}-p\right) \mathbf{n}+\tau \cdot \mathbf{n}\right) \mathrm{d} s
\end{aligned}
$$

A further convenient property of this formulation is that the integration may discard any segments of the contour where the undisturbed flow properties apply, for example the region upstream of the model in the supersonic flow test case.

\section{Test case 1: low-speed bluff-body wake}

\subsection{Experimental procedure}

The first experimental test case considers the highly unsteady vortex wake flow of a square cylinder at incidence. The experiments have been performed in air flow in an open-circuit wind tunnel with a test section of $0.4 \times 0.4 \mathrm{~m}^{2}$ and a low free stream turbulence level of $0.1 \%$. The test model is a cylinder with a square cross section of $30 \times 30 \mathrm{~mm}^{2}$ and a span of $350 \mathrm{~mm}$ (equipped with end plates, for details see Roosenboom 2005), giving a model aspect ratio of 11.7 and area blockage of about $7 \%$. A thin-walled transparent section in the model allows the laser light to pass, permitting the flow around the entire cross-section to be illuminated. Velocity measurements were carried out at different angles of incidence over the range of $0-45^{\circ}$, for a free stream velocity of $10 \mathrm{~m} / \mathrm{s}$. The corresponding value of the Reynolds number based on cylinder diameter $D$ is 20,000 .

The flow was seeded with $1.5 \mu \mathrm{m}$ droplets and illuminated with a Spectra-Physics Quanta-Ray Nd:YAG laser, producing a $2 \mathrm{~mm}$ thick light sheet. The laser wavelength is $532 \mathrm{~nm}$ and the energy is $400 \mathrm{~mJ} /$ pulse, with a pulse duration of $6 \mathrm{~ns}$. A field of view of $135 \times 108 \mathrm{~mm}^{2}(4.5 D \times 3.6 D)$ was imaged on a $1,280 \times 1,024$ pixel CCD camera. A pulse separation of $50 \mu$ s was applied, yielding a maximum particle displacement of 5 pixels. Image analysis was carried out with a window-deformation and iterative multi-grid cross-correlation algorithm (Scarano 2002), using an interrogation window size of $31 \times 31$ pixels and an overlap factor of $75 \%$, yielding a measurement grid with spacing of $0.8 \mathrm{~mm}(0.03 \mathrm{D})$. The spatial resolution is estimated at $1.7 \mathrm{~mm}(0.06 \mathrm{D})$, while the velocity uncertainty is estimated at $2 \%$ of the free stream velocity. The applied image-pair acquisition rate is $3.3 \mathrm{~Hz}$, which does not permit a time-resolved sampling of the shedding phenomenon. Flow statistics were performed with a data ensemble size of about 1,000. For the high-fluctuation levels encountered in the wake (rms up to $100 \%$ of the free stream velocity), this ensemble size corresponds to a mean-flow statistical convergence error of about $3 \%$.

\subsection{Results}

Results of the PIV experiments and resulting pressure and force determination are given in Fig. 2. In Fig. 2a the measured time-mean velocity pattern and derived time-mean pressure field are shown, for the model at zero incidence. Figure $2 b$ displays for this case the computed pressure-gradient vectors, with the separate diagrams displaying the contributions of mean momentum ('Euler terms'), turbulent momentum ('fluctuating terms') and of the viscous stresses, in relation to the different terms in the momentum equation, Eq. 4. The viscous terms are seen to be negligible (note strongly magnified scale), whereas the mean flow terms dominate in the flow outside the wake while the turbulent terms are significant in the wake region. Prescribing the pressure at the inlet side of the domain using Bernoulli's relation, the pressure gradient is integrated by a linewise downstream-marching technique, similar to the procedure described by Bauer and Koengeter (1999).

Figure $2 \mathrm{c}$ synthesizes the final results for the estimated lift and drag of the square cylinder, as a function of incidence angle. The dashed line gives the raw force data as determined in the experiments. The accuracy of the force determination is estimated to result in an error of about \pm 0.1 in the force coefficients, corresponding to $5 \%$ on the actual force value.

To allow comparison to literature data (separate symbols, taken from several sources, see Roosenboom 2005), a correction was applied to account for the model blockage in the wind tunnel test section (Area Blockage Ratio ABR $=7 \%$ ), based on a combination of Maskell's and Newnham's methods:

multiplicative correction factor $=\left(1-0.5 \cdot C_{\mathrm{D}} \cdot \mathrm{ABR}\right)^{2}$

The agreement of the corrected data with literature is quite satisfactory, apart from the drag in the region of $0-10^{\circ}$, where the present drag data are up to $20 \%$ lower. The lower drag value for this model was confirmed by balance measurements (Van Oudheusden 
Fig. 2 Flow around a square section cylinder: a time-mean flow obtained with 2-

component PIV (left, velocity magnitude colour contours) and inferred time-mean pressure distribution (right). b Contribution of different terms to the pressure gradient $\left(\alpha=0^{\circ}\right)$. c Results for the time-mean force coefficients (left: lift, right: drag); red symbols present results (dashed line: not corrected for blockage), other symbols are literature data
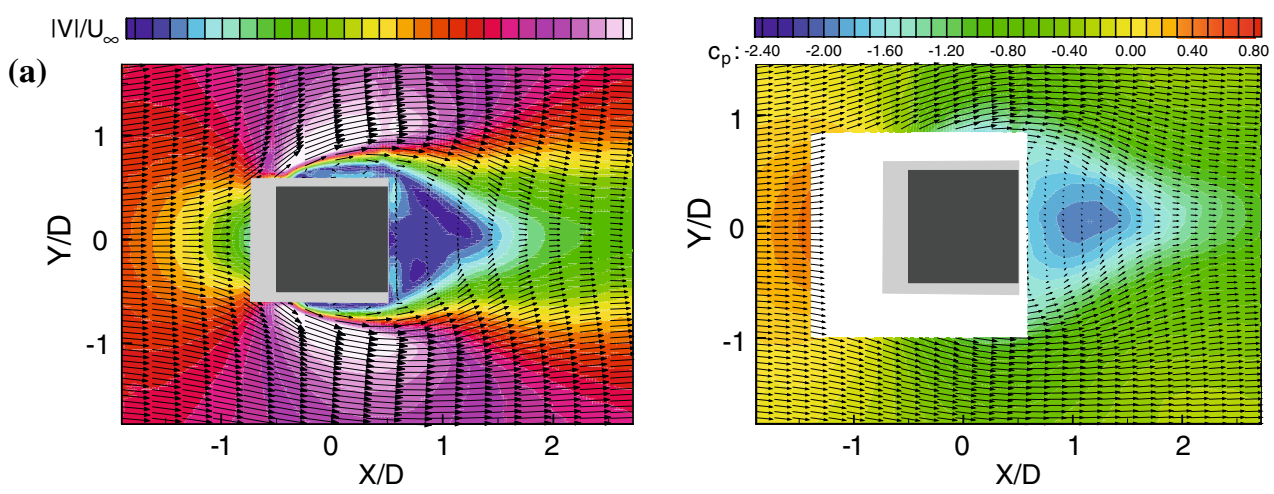

(b)
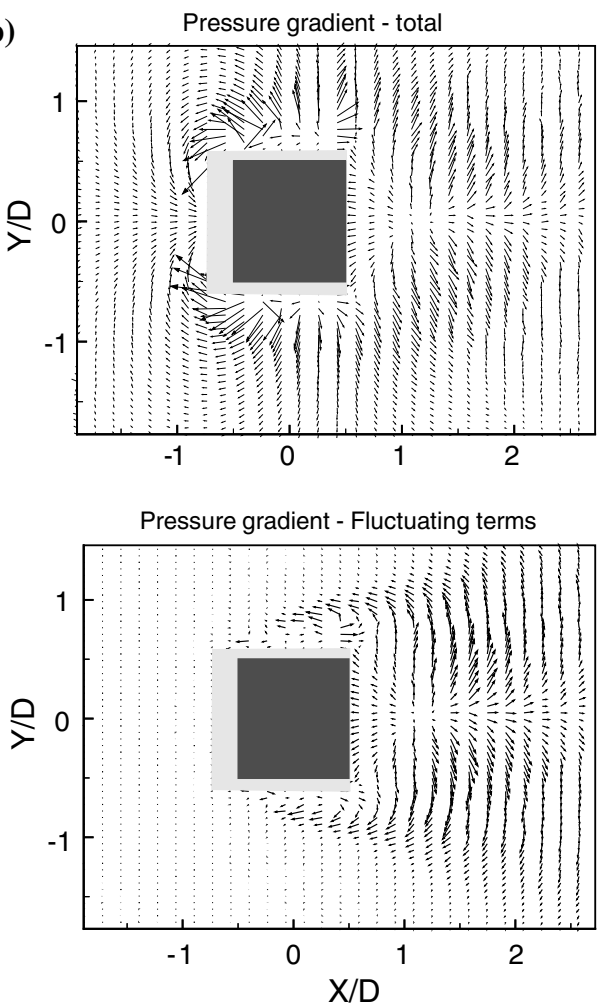

(c)

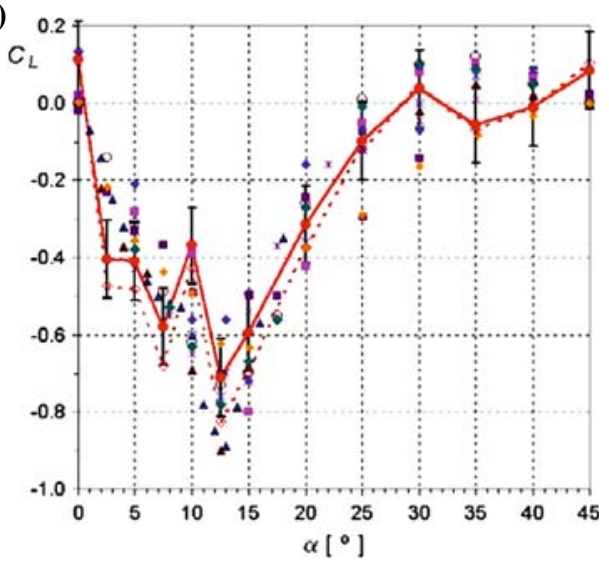

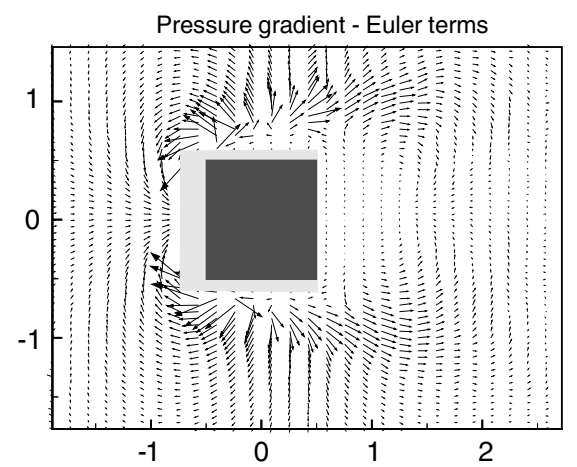

Pressure gradient - Viscous terms (100x magnified)
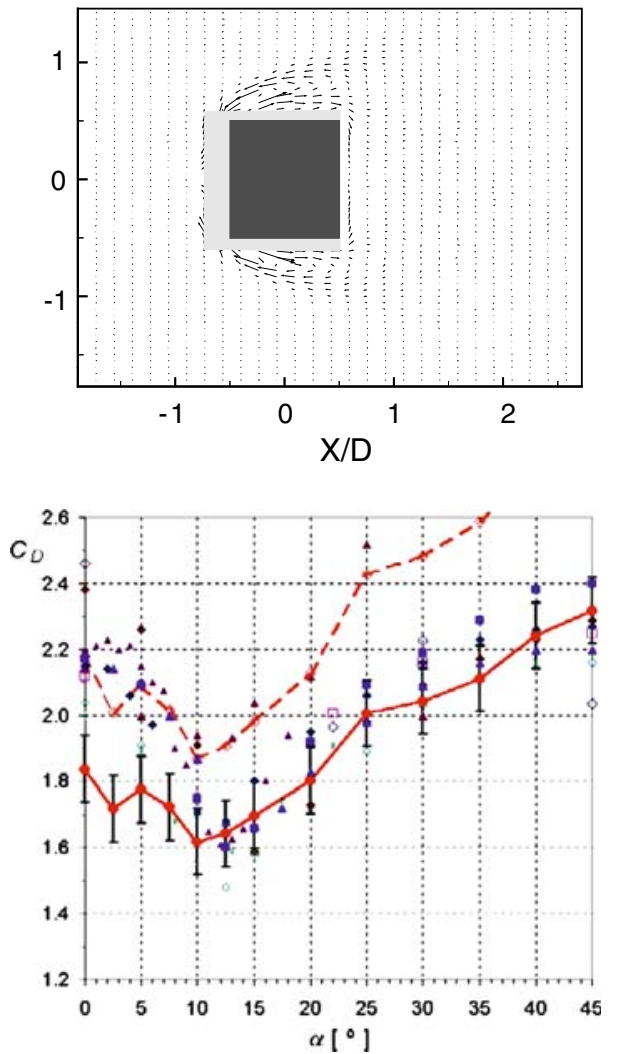
1995), while a similar discrepancy with other studies was found in the length of the wake (Van Oudheusden et al. 2004), which may indicate that possible essential differences in the flow occur for the present model.

\section{Test case 2: low-speed airfoil characterization}

\subsection{Experimental procedure}

The objective of the second experimental test case is to assess the potential of the PIV-based approach for the aerodynamic load characterization of low-speed airfoil sections. Standard procedures for this kind of tests are available and regularly performed at the laboratory, using a verified technique based on pressure measurements. For this the wing model is equipped with a large number of pressure orifices to determine the surface pressure distribution, from which the lift is inferred through integration, while the drag is determined separately using a pitot-tube wake rake at some distance (typically 2-3 chord lengths) behind the airfoil, as described in Jones (1936). The motivation for the present study is to provide a validation of the PIV-based approach with respect to the standard procedures, under realistic wind tunnel conditions. In perspective, the new approach can provide an alternative procedure, notably for low-Reynolds testing, in which case a correct simulation of the Reynolds number would require small dimensions and low flow speeds, which makes pressurebased methods increasingly inaccurate.

A numerical validation was performed, based on synthetic data obtained with a CFD code, to check the PIV-based procedures for consistency. This study verified that lift, drag and pitching moment can be obtained with acceptable accuracy, for details see Van Oudheusden et al. (2006).

The experiments have been performed in the lowspeed low-turbulence wind tunnel, which is a closedcircuit facility with a test section of $1.80 \mathrm{~m} \times 1.25 \mathrm{~m}$ (width $\times$ height). The tests were carried out on a wing model with airfoil section NACA 642A015, with span of $0.64 \mathrm{~m}$ and chord of $0.24 \mathrm{~m}$. The wing was suspended vertically from the upper tunnel wall and equipped at its lower free end with a transparent end plate, which allowed optical access to the flow around the wing from a window in the bottom tunnel wall (Fig. 3). Tests were carried out for a range of incidence angles and with the free stream velocity between 6 and $44 \mathrm{~m} / \mathrm{s}$ (Reynolds number based on chord varies from $100,000$ to 700,000$)$.

For each configuration, tests were performed with the PIV-based technique and with the standard

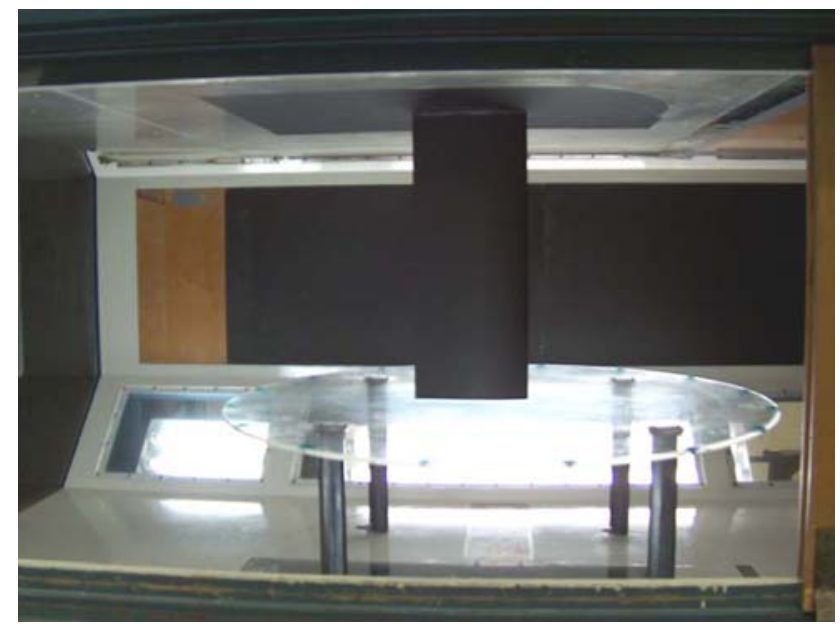

Fig. 3 Experimental setup for PIV-based force characterization of a two-dimensional wing

pressure-based procedures as means of validation. For the PIV experiments, flow seeding and illumination source (laser) were the same as described for the first test case. Illumination was more complex, as the model is non-transparent and also because of the large aspect ratio of the flow field of interest (elongated in streamwise direction). Application of the control volume approach requires the illumination of a complete surrounding of the wing cross section, for which the expanded laser sheet was introduced downstream of the test section and projected onto the model from two mirrors placed on opposite sides of the test section. Laser sheet thickness was about $3 \mathrm{~mm}$. Two CCD camera's $(1,280 \times 1,024$ pixel and 1,376 $\times 1,040$ pixel $)$ were used in a side-by-side configuration to produce an elongated view around the wing cross section, measuring $45 \times 18 \mathrm{~cm}^{2}(1.9 \times 0.75$ chord lengths $)$. An example of the field of view and a typical mean velocity field is displayed in Fig. 4a. Unreliable flow regions, including those obscured by the viewing perspective, have been masked. The pulse separation was chosen such that the free stream velocity corresponds to a particle displacement of 7 pixels. Image analysis was carried out with a window-deformation and iterative multi-grid cross-correlation algorithm, using an interrogation window size of $32 \times 32$ pixels and an overlap factor of $75 \%$, yielding a measurement grid with spacing of ca $1.45 \mathrm{~mm}$ ( $0.6 \%$ chord). For each configuration a data ensemble size of about 100 image pairs was obtained with an acquisition rate of $2 \mathrm{~Hz}$.

\subsection{Results}

Lift, drag and pitching moment coefficient were initially computed by the original control volume method, 
Fig. 4 PIV-based force characterization of a twodimensional wing, experimental results: a mean velocity field result and indication of integration contours. Velocity is in $\mathrm{m} / \mathrm{s}$, free stream velocity is $19 \mathrm{~m} / \mathrm{s}$. b Results for lift, drag and pitching moment versus incidence angle; comparison between PIV results and pressure data

(a)

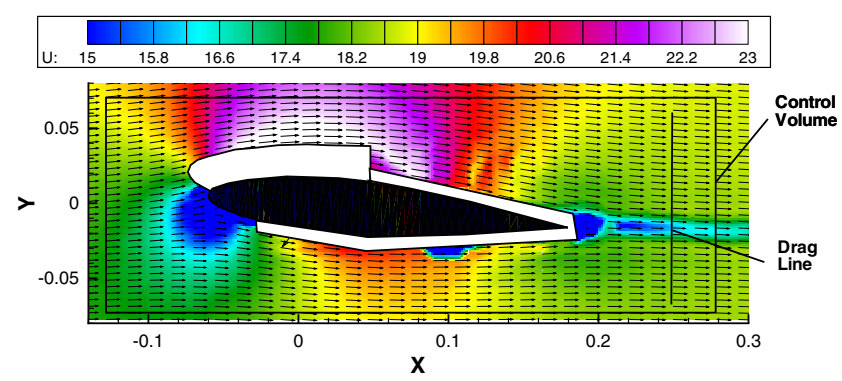

(b)

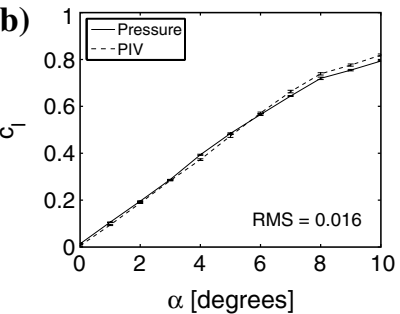

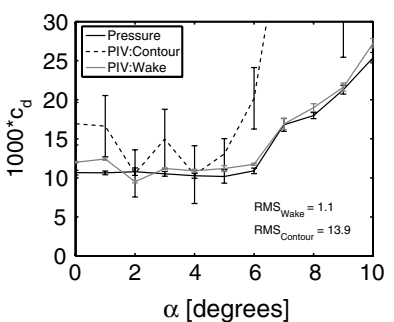

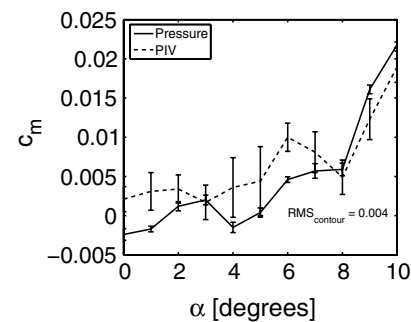

Eq. 3, taking a contour around the wing as illustrated in Fig. 4a. The uncertainty estimate of the load values was based on changing the size of the contour, varying the distance to the wing between 0.35 and 0.5 chord lengths. In view of the low value of the drag, application of the contour procedure yielded unacceptable errors, and the drag-determination procedure is much improved by introducing a classical wake approach instead. Here the static pressure is calculated along a vertical line across the wake (see Fig. 4a), which allows computation of the total pressure coefficient $c_{\mathrm{pt}}$. The drag is then determined according to Jones (1936), as:

$D=\rho U_{\infty} \int_{\text {wake }} u\left(1-\sqrt{c_{\mathrm{pt}}}\right) \mathrm{d} y$

This approach can be seen as a particular implementation of the mass-conservation correction discussion in Sect. 2.3, and the above expression is accepted as being valid even close to the trailing edge. It may be noted that the location of the wake traverse in the PIV approach is much closer to the airfoil trailing edge $(0.5 \mathrm{c}$ max) than commonly applied for a wake rake in view of the latter's possible intrusive effect on the flow. Changing the location of this line (between 0.25 and 0.5 chord lengths behind the wind) again provides an estimate of the drag uncertainty.

Variations of the lift and drag as function of incidence angle are given in Fig. 4b, for $R e=300,000$. The uncertainty range of the data is indicated by the error bars (corresponding to \pm two standard deviations). Mean differences (in the rms sense) between PIV and pressure based data, for the flow conditions considered, are 0.016 on the lift coefficient, $1 \times 10^{-3}$ on the drag coefficient using the wake approach $\left(14 \times 10^{-3}\right.$ with the contour approach) and $4 \times 10^{-3}$ for the moment coefficient.

In conclusion, the comparison between the PIVbased force coefficients and the standard pressurebased procedure clearly demonstrates the capability of the PIV method to provide a non-intrusive characterization of the wing, based on velocity field information.

\section{Test case 3: bi-convex airfoil in supersonic flow}

The third test case considers the extension of the PIVbased approach for aerodynamic load characterization to the high-speed flow regime. The aim was to develop a load determination method for flows with variable density, with the possible occurrence of shock waves as an additional complication. This is regarded a very interesting and essential extension of this technique since many wing-related applications concern the compressible flight regime. Further, from the facility point of view, this technique would permit the determination of aerodynamic loads (lift and drag) in highspeed wind tunnels that are not equipped with a force balance.

Shock waves require special attention in the loads and pressure determination as they represent (near-) discontinuous features of the flow field. Moreover, the velocity field in their direct vicinity cannot be resolved properly by the PIV method, due to finite particle inertia and optical imaging distortion effects (Elsinga et al. 2004). The treatment of shocks applied in the present procedure is to first identify the shocks (position and inclination, hence, strength) from the velocity 
field, by means of an interactive graphical procedure. Subsequently, the shock regions are masked and the pressure integration is propagated over the shocks using theoretical shock relations (Souverein 2006).

\subsection{Procedure validation}

The data analysis procedures were also in this case validated by means of synthetic flow data, addressing the inviscid flow around a diamond wedge airfoil (Fig. 5), for which the exact flow solution is available from shock wave expansion theory. Some variation of the free stream Mach number and incidence angle was considered. A sensitivity analysis was made with respect to several parameters, including the pressure integration strategy, the input for the shock locations, noise on the velocity field, uncertainties in the free-stream quantities, and the influence of the data resolution.

(a)
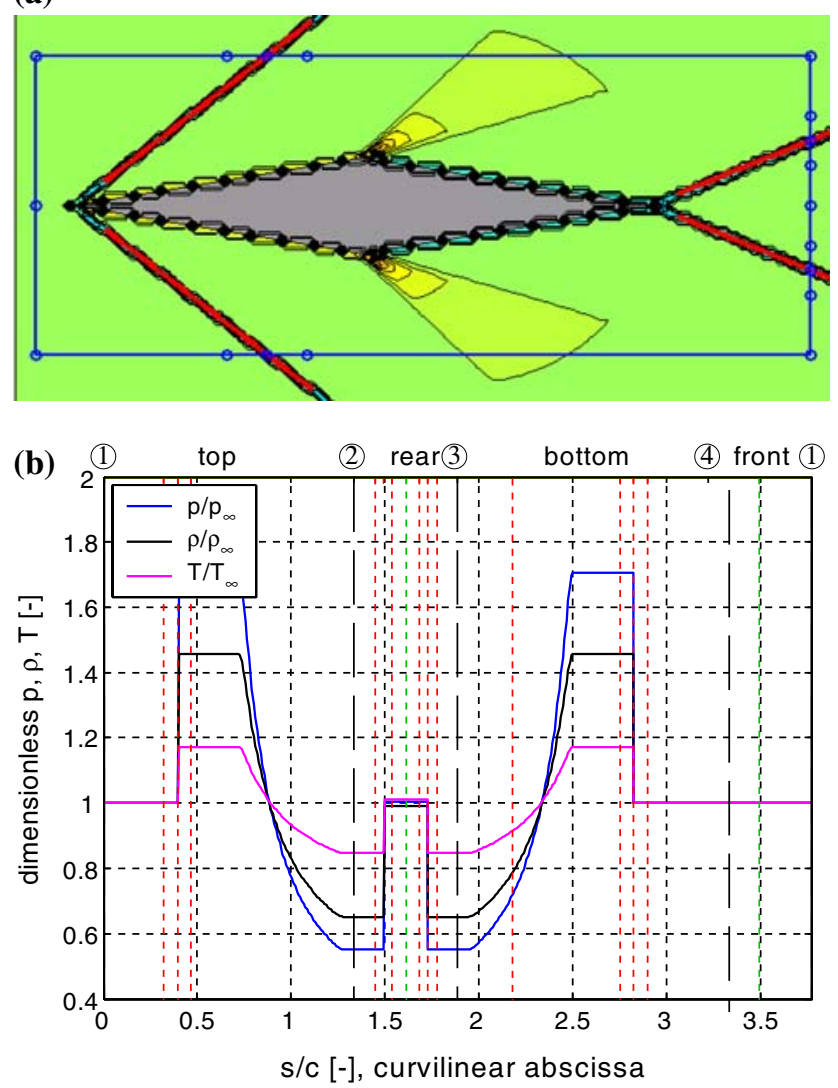

Fig. 5 PIV-force characterization of an airfoil in supersonic flow, synthetic test case: theoretical simulation of the inviscid flow field around a diamond-shaped airfoil. a Velocity divergence field (colours), definition of shock locations (red), integration contour (blue). b Results for pressure, density and temperature along the contour $\left(M=2, \alpha=0^{\circ}\right)$
Figure 5a illustrates the procedure of identifying the shocks, based on the divergence of the velocity field, and identifies the integration contour and shock masking regions. The integration results for a typical case are depicted in Fig. 5b, showing the variation of pressure, density and temperature along the integration contour.

The results of the simulations confirm that the integration of velocity data to obtain pressure and aerodynamic loads is feasible, also for compressible flows with shocks. The current approach, based on a contour integration of Eq. 6 for the pressure, and a masking and modeling approach for the traversal of shocks with a user input for the shock location, yields good results. In the ideal case, using the theoretical flow properties as input, the error in the lift and drag was 0.06 and $0.2 \%$, respectively. The sensitivity analysis shows the load prediction to be most affected by the user input of the shock coordinates and by the noise in the velocity field. It is concluded that for realistic input data, none of the individual parameters prohibits a viable lift and drag estimate. It was also found that a split path integration approach (with one half following the upper branch of the contour and the other half following the lower branch) yields significantly better and more consistent results than a single integration along the complete contour. The investigation further revealed that the accuracy of the drag integration is mostly determined by the momentum flux contribution and that of the lift integration by the pressure term. Furthermore, it was found that the corrected formulation of Eq. 7 that compensates for mass defect over the contour, increases the accuracy of the drag determination by an order of magnitude as compared to the original contour integral approach of Eq. 1. With user input for the shocks, errors in the order of $1 \%$ or less are then achieved for both the lift and the drag. Imposing noise on the velocity field yields lift and drag errors of around 5 and $2 \%$, respectively for a representative random noise level of $0.2 \%$ of the freestream velocity. Errors of about 40 and $20 \%$ are obtained for the lift and drag respectively for a random noise of $1 \%$ of the freestream velocity.

\subsection{Experimental verification}

Experiments were performed in the TST-27 blowdown transonic-supersonic wind tunnel; test section dimensions are $280 \times 255 \mathrm{~mm}^{2}$ (width $\times$ height). Tests were carried out on a full span bi-convex airfoil with a chord of $100 \mathrm{~mm}$ and a thickness of $12 \mathrm{~mm}$. Incidence angles were taken in the range of $-6^{\circ}$ to $6^{\circ}$ and two free stream Mach numbers (2.0 and 2.5) were considered. 
The corresponding Reynolds number based on the chord is $2.7 \times 10^{6}$ and $3.4 \times 10^{6}$, respectively. The model was equipped with pressure orifices, allowing for a surface pressure based estimate of the aerodynamic loading (lift and drag). From flat plate theory it was estimated that the viscous drag is of the order of a percent of the profile drag and may be neglected in this evaluation. A further verification of the measured loads was performed using Burgers theory. The difference with the pressure-based results was in the order of $1-10$ and $1 \%$ for the lift and the drag, respectively.

For the PIV measurements, the flow was seeded with $400 \mathrm{~nm} \mathrm{TiO}_{2}$ particles. Illumination was provided by a Big Sky Laser CFR PIV-200 Nd:YAG laser, producing a $1.5 \mathrm{~mm}$ thick light sheet. The laser wavelength is $532 \mathrm{~nm}$ and the energy is $200 \mathrm{~mJ} /$ pulse, with a pulse duration of $6 \mathrm{~ns}$. A field of view of $146.5 \times 80.1 \mathrm{~mm}^{2}$ was imaged with a $1,280 \times 1,024$ (vertical image size cropped to 800) pixel CCD camera (see Fig. 6a). With illumination from behind, a small shadow region upstream of the model occurs, which does not hinder the force determination when choosing the contour such that it crosses the shadow in the free stream flow upstream of the attached bow shocks. A pulse separation of 3 ( $\mathrm{s}$ was applied, yielding a maximum particle displacement of 15 pixels. Image analysis was carried out with the same software as described in the first test case. A window size of $31 \times 31$ pixels was applied with an overlap of $75 \%$, yielding a measurement grid with spacing of about $0.9 \mathrm{~mm}(0.9 \%$ chord $)$. For each configuration, a data ensemble size of about 200 image pairs was obtained with an acquisition rate of $4.2 \mathrm{~Hz}$.

The experimental results (Fig. 6b) confirm that force characterization from PIV velocity data is possible also under these flow conditions. The shock identification and modeling method based on a user input works adequately. Furthermore, consistent results are obtained for the loads at all angles of attack. The error bars in the graphs represent the uncertainty related to the manual shock input. Similar accuracy is obtained as for the numerical flow field with a representative noise level on the velocity field. The mean deviation (rms) between PIV and pressure based data are 0.033 on the lift coefficient and 0.013 on the drag coefficient, for the flow conditions considered (corresponding to approximately $16 \%$ of the full scale values). There appears to be a systematic bias in the drag determination for negative incidence, the reason for this is not known.

In conclusion, the current experimental investigation, supported by the numerical validation, clearly demonstrates the capability of the PIV method to provide a non-intrusive loads characterization in the
Fig. 6 PIV-force

characterization of an airfoil in supersonic flow,

experiment: bi-convex airfoil: a mean velocity field $(M=2$; $\left.\alpha=0^{\circ}\right)$. b Lift and drag versus incidence angle; comparison between PIV results and pressure data

(a)

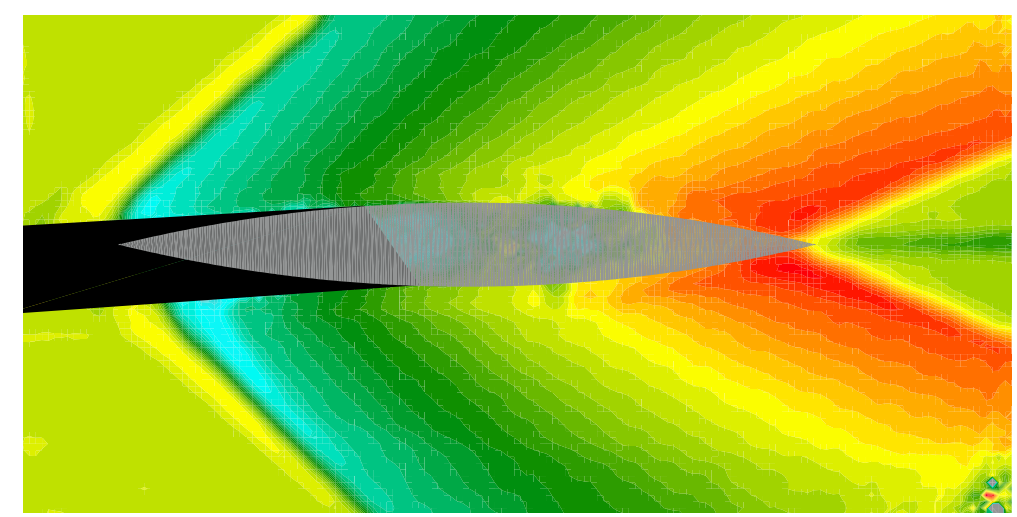

(b)

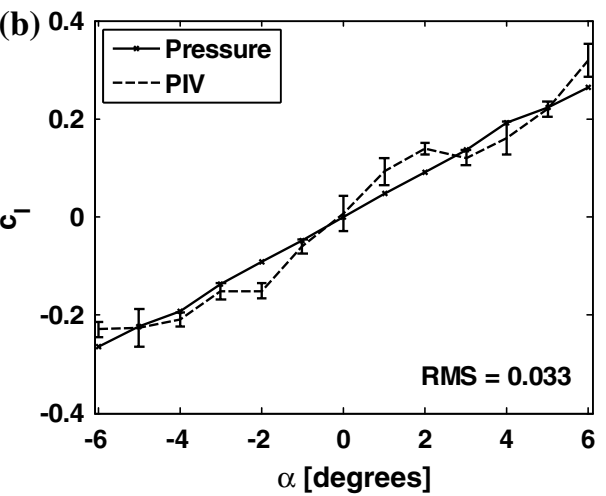

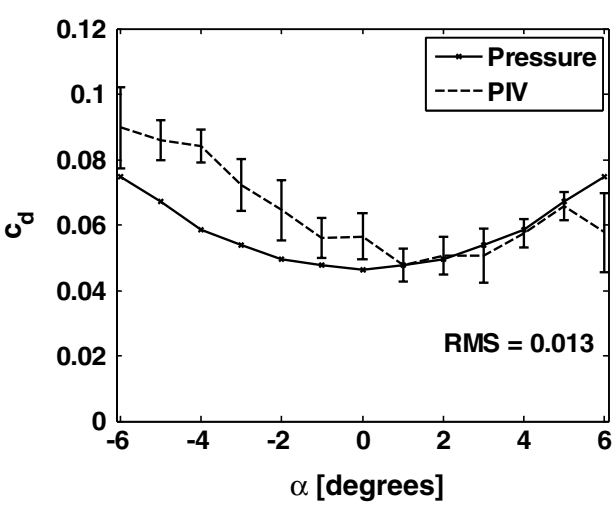


considered supersonic flow regime. It may be added that the drag estimate proved only viable by using the corrected contour integration according to Eq. 7; with use of the original contour expression no reliable drag estimate resulted (errors of $100 \%$ or more).

\section{Conclusions}

The approach to determine pressure fields and integral loads from planar velocimetry data was considered as a means for non-intrusive aerodynamic load characterization, for incompressible and compressible flows around two-dimensional objects. The method relies upon the application of control-volume approaches in combination with the deduction of the pressure field from the experimental data, by making use of the flow constitutive equations. The implementation for two specific application areas was addressed.

The first is time-mean pressure field and integral force evaluation from velocity ensemble statistics, as obtained from time-uncorrelated PIV acquisition, for low speed (incompressible) flow. Two test cases are considered: the unsteady flow around a square section cylinder at incidence, as well as the force characterization of a low-speed airfoil. In the first case, which is a highly unsteady flow, velocity statistics were based on ensemble size of 1,000 vector fields. The resulting confidence on the non-dimensional force coefficients in these experiments is estimated to be about 0.1. For the airfoil test case pressure-based results were available which allowed the PIV method to be validated. With the flow being predominantly steady, an ensemble size of 100 turned out to be sufficient to produce force data with sufficient accuracy. The lift was determined from the contour approach, while for the drag a wake-section approach was found to improve accuracy significantly.

The second topic considers the extension of the method to steady compressible flow. As in this flow regime the density appears as an extra unknown in the momentum equation, additional flow equations need to be invoked. A convenient approach for this was found, using the gas law and the adiabatic flow condition, with which the pressure-integration procedure becomes essentially the same as for the incompressible case. In the specific application of supersonic flow, an additional complication is posed by the occurrence of shock waves. Again, it was found that the drag estimate was much improved by correcting the original control volume expression for mass flux deficit.

\section{References}

Adrian RJ (2005) Twenty years of particle image velocimetry. Exp Fluids 39:159-169

Anderson JD Jr (1991) Fundamentals of aerodynamics, 2nd edn. McGraw-Hill, New York

Bauer T, Koengeter J (1999) PIV with high temporal resolution for the determination of local pressure reductions from coherent turbulent phenomena. In: 3rd International workshop on PIV, Santa Barbara, pp 671-676

Berton E, Maresca C, Favier D (2004) A new experimental method for determining local airloads on rotor blades in forward flight. Exp Fluids 37:455-457

Brune GW (1994) Quantitative low-speed wake surveys. J Aircraft 31:249-255

Elsinga GE, van Oudheusden BW, Scarano F (2004) Evaluation of optical distortion effects in PIV. Exp Fluids 39:246-256

Fujisawa N, Tanahashi S, Srinavas K (2005) Evaluation of pressure field and fluid forces on a circular cylinder with and without rotational oscillation using velocity data from PIV measurement. Mea Sci Techn 16:989-996

Gurka R, Liberzon A, Hefetz D, Rubinstein D, Shavit U (1999) Computation of pressure distribution using PIV velocity data. In: 3rd International workshop on PIV, Santa Barbara, pp 101-106

Hosokawa S, Moriyama S, Tomiyama A, Takada N (2003) PIV measurement of pressure distributions about single bubbles. J Nuclear Sci Techol 40:754-762

Jones BM (1936) Measurement of profile drag by the PitotTraverse method. ARC R\&M 1688

Lin JC, Rockwell D (1996) Force identification by vorticity fields: techniques based on flow imaging. J Fluids Struct 10:663-668

Noca F, Shiels D, Jeon D (1999) A comparison of methods for evaluating time-dependent fluid dynamic forces on bodies, using only velocity fields and their derivatives. J Fluids Struct 13:551-578

Raffel M, Willert C, Kompenhans J (1998) Particle Image Velocimetry, a practical guide. Springer, Heidelberg

Roosenboom EWM (2005) Experimental analysis of the flow around a cylinder with a square cross-section. MSc Thesis, Delft University of Technology, Department of Aerospace Engineering

Scarano F (2002) Iterative image deformation methods in PIV. Mea Sci Technol 11:R1-R19

Souverein LJ (2006) PIV based aerodynamic loads determination in supersonic flows. MSc Thesis, Delft University of Technology, Department of Aerospace Engineering

Unal MF, Lin JC, Rockwell D (1997) Force prediction by PIV imaging: a momentum based approach. J Fluids Struct 11:965-971

Van Oudheusden BW (1995) On the quasi-steady analysis of one-degree-of-freedom galloping with combined translational and rotational effects. Nonlin Dyn 8:435-451

Van Oudheusden BW, Scarano F, Van Hinsberg NP, Manna L (2004) Quantitative visualization of the flow around a square-section cylinder at incidence. In: 5th Int. Colq. Bluff Body Aero. \& Appl., Ottawa, pp 189-192

Van Oudheusden BW, Scarano F, Casimiri EWF (2006) Nonintrusive load characterization of an airfoil using PIV. Exp Fluids 40:988-992

White FM (1991) Viscous fluid flow, 2nd edn. McGraw-Hill, New York

Zhu G, Bearman PW, Graham JMR (2002) Prediction of drag and lift using velocity and vorticity fields. Aeron J 106:547-554 